\title{
SACCoM 20 años
}

\section{Reseña}

\section{Mirian Tuñez' y Guadalupe Segalerba² mirtun55@gmail.com}

\author{
Laboratorio para el Estudios de la Experiencia Musical - Facultad de Artes \\ Universidad Nacional de La Plata
}

\section{Resumen}

Este artículo relata la creación e ideas fundacionales de la Sociedad Argentina para las Ciencias Cognitivas de la Música (SACCoM). Congrega a dos de sus socios fundadores: Isabel Cecilia Martínez y Favio Shifres quienes evocan los principios que le otorgan identidad y regionalidad al estudio de la experiencia musical desarrollada en los contextos socioculturales en los que tiene lugar. Destacan a su vez, la difusión y promoción del conocimiento musical desde una perspectiva plural e interdisciplinar como posicionamiento de base en el desarrollo de la investigación.

\section{Palabras Clave}

experiencia musical, SACCoM, producción de conocimiento. 


\title{
SACCOM 20 years
}

Review

\begin{abstract}
This article reports the creation and founding ideas of the Argentine Society for Cognitive Sciences of Music (SACCoM). It brings together two of its founding partners: Isabel Cecilia Martínez and Favio Shifres who evoke the principles that give identity and regionality to the study of musical experience developed in the socio-cultural contexts in which it takes place. And they stand out in turn, the dissemination and promotion of musical knowledge from a plural and interdisciplinary perspective as a basic position in the development of research.
\end{abstract}

\section{Key Words}

music experience, SACCoM, knowledge promotion..

La Sociedad Argentina para las Ciencias Cognitivas de la Música (SACCoM) nace con el siglo XXI. Fundada por Isabel Cecilia Martínez, Favio Shifres y Orlando Musumeci con el objetivo de difundir y promover el conocimiento musical desde una mirada interdisciplinaria, impulsando el desarrollo de la investigación, SACCoM favoreció desde sus inicios la cooperación entre docentes e investigadores, la formación de estudiantes y el apoyo a jóvenes investigadores. Así mismo, sostiene la comunicación entre la comunidad de educadores, estudiantes e investigadores de nuestra región (particularmente de los países de habla hispana y portuguesa), vinculándose con otras organizaciones nacionales e internacionales que comparten sus principales objetivos. La Sociedad también ha tenido un gran impacto en la conformación de otras agrupaciones similares, tales como la Asociación Colombiana de Investigadores en Psicología de la Música y Educación Musical, y la Asociación Brasileña de la Cognición y las Artes Musicales.

Con el fin de alcanzar sus objetivos, SACCoM convoca y reúne organizando de manera sistemática encuentros y jornadas académicas en diferentes puntos del país, tanto en universidades nacionales como en institutos terciarios provinciales, y con asidua participación de asistentes locales e internacionales. Desde su editorial, difunde con libre acceso las actas de las jornadas mencionadas y edita la revista Epistemus con frecuencia semestral, gracias al trabajo sostenido de editores y evaluadores que apuestan a las publicaciones en español. Se suma además el 
apoyo del Laboratorio para el Estudio de la Experiencia Musical (LEEM) de la Facultad de Artes de la Universidad Nacional de La Plata, cuyos integrantes son miembros de SACCoM.

\section{Reuniones y encuentros}

Las primeras reuniones se desarrollaron en la provincia de Buenos Aires -en las ciudades de Avellaneda, Quilmes y La Plata- con invitados como John Sloboda, Ian Cross y Michel Imberty. Luego los encuentros se sucedieron en Tucumán, Corrientes, Entre Ríos, Santa Fe, Córdoba, San Juan, la Ciudad Autónoma de Buenos Aires y nuevamente en la Provincia de Buenos Aires, recorriendo las ciudades de Bahía Blanca y La Plata (Figura 1). Así, a lo largo de estos 20 años fueron invitados investigadores de diferentes disciplinas a dictar conferencias centrales y participar de mesas redondas. En el plano internacional recordamos, además de los ya mencionados, la presencia de Florentino Blanco, Graham Welch, Rubén López Cano, Antoni Gomila, Luis Naveda, Ellen Dissanayake y Cintia Rodriguez. Y en el plano nacional a Héctor Rubio, Omar Corrado, y Enrique Piñeyro.

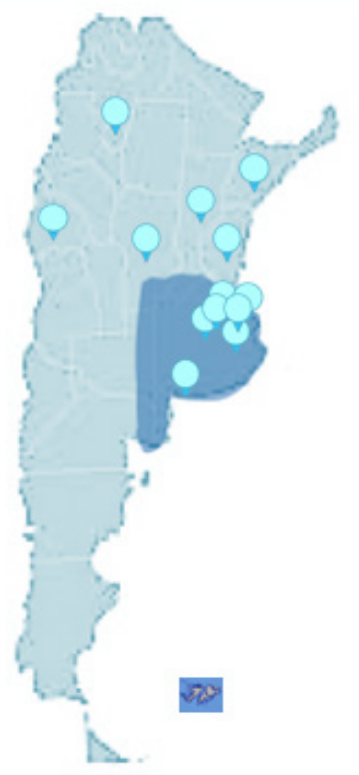

Figura 1. Ubicación geográfica de los encuentros. 
Las instituciones anfitrionas fueron diferentes universidades nacionales, escuelas de música, institutos superiores y conservatorios, así como también el Espacio Memoria y Derechos Humanos. De estas reuniones y encuentros participaron alumnos, docentes e investigadores de nuestro país y de la región.

Los temas convocantes se estructuraron con relación a los procesos implicados en la experiencia musical, el rol de la cultura y la educación en el desarrollo de la cognición musical; los avances desde la psicología cognitiva, del desarrollo y la comunicación; las prácticas musicales en el marco de las ciencias cognitivas de la música; la música y el bienestar humano; la objetividad, la subjetividad y la música; la experiencia artística y la cognición musical; la tradición y la diversidad en los aspectos psicológicos, socioculturales y musicológicos de la formación musical; la musicalidad humana, los debates actuales en evolución, desarrollo, cognición e implicancias socio-culturales; nuestro cuerpo en nuestra música; la experiencia musical: el cuerpo, el tiempo y el sonido en el escenario de nuestra mente; la experiencia musical: investigación, interpretación y prácticas educativas. La participación de asistentes y expositores de 14 universidades públicas argentinas y 14 universidades latinoamericanas en el último encuentro presencial $\left(13^{\circ} \mathrm{ECCoM}\right.$ 2017) realizado en la ciudad de La Plata, es una clara muestra del alcance de los eventos organizados por la Sociedad.

Este último año hemos asistido a la organización del Ciclo de Conversatorios 2020, en modalidad virtual y difundido vía Zoom y streaming, cuyos cinco encuentros están aún disponibles con libre acceso en el sitio web de SACCoM. Diferentes trabajos vinculados a la experiencia musical en relación con la colonialidad del saber, las aproximaciones metodológicas a su estudio, la segunda persona y las experiencias de feminismo en las prácticas sonoras y musicales, fueron presentados y debatidos con el aporte de especialistas argentinos en psicología de la música y en ciencias sociales. Las visualizaciones del evento se dieron desde América y Europa (Tabla 1).

\section{Momentos de la historia de SACCoM en primera persona}

Los doctores Favio Shifres e Isabel Cecilia Martínez son dos de los referentes que fundaron la sociedad y la acompañaron en carácter de presidentes. Ambos dirigen hoy sus equipos de investigación en la Facultad de Artes de la Universidad Nacional de La Plata como docentes titulares. Con motivo de los 20 años de SACCoM, nos comparten sus recuerdos y reflexiones en el marco de una charla generosa y emotiva. 
Epistemus - volumen 8 - número 2 (Diciembre de 2020)

DOI: https://doi.org/10.24215/18530494e024

\begin{tabular}{|c|c|c|c|c|}
\hline 1ra Reunión & $\begin{array}{l}\text { La música en la mente. } \\
\text { Procesos implicados en la } \\
\text { experiencia musical. }\end{array}$ & $\begin{array}{c}\text { Facultad de Psicología } \\
\text { de la Universidad de } \\
\text { Buenos Aires. }\end{array}$ & $\begin{array}{l}\text { Avellaneda, Pro- } \\
\text { vincia de Buenos } \\
\text { Aires. }\end{array}$ & $\begin{array}{c}2001 \\
5 \text { de mayo }\end{array}$ \\
\hline 2da Reunión & Temáticas Abiertas. & $\begin{array}{l}\text { Universidad Nacional de } \\
\text { Quilmes. }\end{array}$ & $\begin{array}{l}\text { Quilmes, Provincia } \\
\text { de Buenos Aires. }\end{array}$ & $\begin{array}{c}2002 \\
19 \text { y } 20 \text { de abril }\end{array}$ \\
\hline 3ra Reunión & $\begin{array}{l}\text { El rol de la Cultura y la Edu- } \\
\text { cación en el Desarrollo de la } \\
\text { Cognición Musical. }\end{array}$ & $\begin{array}{c}\text { Rectorado de la } \\
\text { Universidad Nacional de } \\
\text { La Plata. }\end{array}$ & $\begin{array}{l}\text { La Plata, Provincia } \\
\text { de Buenos Aires. }\end{array}$ & $\begin{array}{c}2003 \\
11 \text { y } 12 \text { de abril }\end{array}$ \\
\hline 4ta Reunión & $\begin{array}{c}\text { Avances desde la Psicología } \\
\text { Cognitiva, del Desarrollo y la } \\
\text { Comunicación. }\end{array}$ & $\begin{array}{l}\text { Instituto Superior de Mú- } \\
\text { sica de la Universidad } \\
\text { Nacional de Tucumán. }\end{array}$ & $\begin{array}{l}\text { Provincia de } \\
\text { Tucumán. }\end{array}$ & $\begin{array}{c}2004 \\
14 \text { y } 15 \text { de } \\
\text { mayo }\end{array}$ \\
\hline 5ta Reunión & $\begin{array}{l}\text { Las Prácticas Musicales en } \\
\text { el Marco de las Ciencias } \\
\text { Cognitivas de la Música }\end{array}$ & $\begin{array}{c}\text { Instituto Superior de } \\
\text { Música Prof. Carmelo H. } \\
\text { De Biasi. }\end{array}$ & $\begin{array}{l}\text { Provincia de Co- } \\
\text { rrientes. }\end{array}$ & $\begin{array}{c}2006 \\
1 \text { al } 3 \text { de junio }\end{array}$ \\
\hline 6ta Reunión & Música y bienestar humano. & $\begin{array}{c}\text { Facultad de Humanida- } \\
\text { des, Artes y Ciencias } \\
\text { Sociales de la Universi- } \\
\text { dad Autónoma de Entre } \\
\text { Ríos. }\end{array}$ & $\begin{array}{l}\text { Concepción del } \\
\text { Uruguay, Provincia } \\
\text { de Entre Ríos. }\end{array}$ & $\begin{array}{c}2007 \\
6 \text { y } 7 \text { de abril }\end{array}$ \\
\hline 7ma Reunión & $\begin{array}{l}\text { Objetividad, Subjetividad y } \\
\text { Música. }\end{array}$ & $\begin{array}{c}\text { Facultad de Humani- } \\
\text { dades y Artes de la } \\
\text { Universidad Nacional de } \\
\text { Rosario. }\end{array}$ & $\begin{array}{l}\text { Rosario, Provincia } \\
\text { de Santa Fe. }\end{array}$ & $\begin{array}{c}2008 \\
3 \text { y } 4 \text { de julio }\end{array}$ \\
\hline 8va Reunión & $\begin{array}{c}\text { La Experiencia Artística y la } \\
\text { Cognición Musical. }\end{array}$ & $\begin{array}{c}\text { Universidad Nacional de } \\
\text { Villa María. }\end{array}$ & $\begin{array}{c}\text { Ciudad de Villa } \\
\text { María, Provincia de } \\
\text { Córdoba. }\end{array}$ & $\begin{array}{l}2009 \\
25 \text { y } 16 \text { de } \\
\text { junio }\end{array}$ \\
\hline 9na Reunión & $\begin{array}{l}\text { Tradición y diversidad en los } \\
\text { aspectos psicológicos, socio- } \\
\text { culturales y musicológicos de } \\
\text { la formación musical. }\end{array}$ & $\begin{array}{l}\text { Conservatorio de Músi- } \\
\text { ca de Bahía Blanca. }\end{array}$ & $\begin{array}{l}\text { Ciudad de Bahía } \\
\text { Blanca, Provincia } \\
\text { de Buenos Aires. }\end{array}$ & $\begin{array}{l}2010 \\
14 \text { y } 15 \text { de } \\
\text { mayo }\end{array}$ \\
\hline 10mo Encuentro & $\begin{array}{l}\text { Musicalidad Humana. Deba- } \\
\text { tes Actuales en Evolución, } \\
\text { Desarrollo, Cognición e Im- } \\
\text { plicancias Socio-Culturales. }\end{array}$ & $\begin{array}{l}\text { Universidad Abierta } \\
\text { Interamericana. }\end{array}$ & $\begin{array}{l}\text { Ciudad Autónoma } \\
\text { de Buenos Aires. }\end{array}$ & $\begin{array}{l}2012 \\
20 \text { al } 23 \text { de } \\
\text { julio }\end{array}$ \\
\hline 11vo Encuentro & $\begin{array}{l}\text { Nuestro Cuerpo en Nuestra } \\
\text { Música. }\end{array}$ & $\begin{array}{l}\text { Espacio Memoria y } \\
\text { Derechos Humanos. }\end{array}$ & $\begin{array}{l}\text { Ciudad Autónoma } \\
\text { de Buenos Aires. }\end{array}$ & $\begin{array}{c}2013 \\
12 \text { al } 14 \text { de } \\
\text { septiembre } \\
\end{array}$ \\
\hline 12vo Encuentro & $\begin{array}{c}\text { La Experiencia Musical: } \\
\text { cuerpo, tiempo y sonido } \\
\text { en el escenario de nuestra } \\
\text { mente. }\end{array}$ & $\begin{array}{l}\text { Universidad Nacional de } \\
\text { San Juan. }\end{array}$ & $\begin{array}{l}\text { Provincia de San } \\
\text { Juan. }\end{array}$ & $\begin{array}{l}2015 \\
13 \text { al } 15 \text { de } \\
\text { agosto }\end{array}$ \\
\hline 13vo Encuentro & $\begin{array}{l}\text { La Experiencia Musical: } \\
\text { investigación, interpretación } \\
\text { y prácticas educativas. }\end{array}$ & $\begin{array}{l}\text { Conservatorio de Músi- } \\
\text { ca Gilardo Gilardi. }\end{array}$ & $\begin{array}{l}\text { La Plata, Provincia } \\
\text { de Buenos Aires. }\end{array}$ & $\begin{array}{c}2017 \\
28 \text { al } 30 \text { de } \\
\text { septiembre }\end{array}$ \\
\hline $\begin{array}{c}\text { Ciclo de } \\
\text { Conversatorios }\end{array}$ & $\begin{array}{l}\text { La Colonialidad del Saber en } \\
\text { la Experiencia Musical. } \\
\text { La Segunda Persona y la } \\
\text { Música. } \\
\text { Aproximaciones Metodo- } \\
\text { lógicas al Estudio de la } \\
\text { Experiencia Musical. } \\
\text { Experiencias de Feminismo } \\
\text { en Prácticas Sonoras y } \\
\text { Musicales. }\end{array}$ & SACCOM & $\begin{array}{c}\text { Modalidad Virtual. } \\
\text { Transmitido desde } \\
\text { la Ciudad de La } \\
\text { Plata } \\
\text { vía Zoom y Strea- } \\
\text { ming. }\end{array}$ & $\begin{array}{c}2020 \\
21 \text { de agosto } \\
25 \text { de sep- } \\
\text { tiembre } \\
16 \text { y } 30 \text { de } \\
\text { octubre } \\
13 \text { de noviem- } \\
\text { bre }\end{array}$ \\
\hline
\end{tabular}

Tabla 1. Detalle de los eventos organizados por la Sociedad. 
Sabemos así, que la idea de crear la Sociedad nació con las primeras reflexiones acerca de la naturaleza de la experiencia musical, en la búsqueda de respuestas a interrogantes sobre su estudio y su enseñanza. Para materializar y consolidar este espacio de reflexión e intercambio en América del Sur, era necesario un respaldo institucional.

"Habíamos ido a la 6ta ICMPC, la conferencia internacional de percepción y cognición musical en Keele, Inglaterra. Cuando termina la conferencia, se hace una reunión plenaria como fin del congreso y la tradición de esas conferencias era que cada una estaba a cargo de una asociación distinta. Eran tres asociaciones: la japonesa, la europea y la norteamericana. Ésta había sido la europea, la anterior había sido en Corea, zona de influencia de Japón y la siguiente estaba programada en la zona del Pacífico, en Sidney. Había que programar la que seguía, o sea la 8va ICMPC, que debía ser en América. Entonces Gabrielsson -que presidía la Conferencia- dice: "bueno, tenemos que elegir un próximo lugar, esperamos recibir propuestas, recordemos que tiene que ser en América". Entonces nos mira a nosotros y nos dice: pero, por supuesto que no tiene por qué ser en Norteamérica... y ahí nos entusiasmó con la idea de hacer la conferencia. Lo primero que dijimos fue que era necesario darle un marco institucional, crear una asociación que le diera soporte a la idea." Recuerda Favio Shifres.

A partir de esta motivación, y con un gran entusiasmo por hacer posible la concreción del nuevo organismo, surgió la necesidad de diseñar un modelo organizativo capaz de formalizar encuentros que promuevan la comunicación y la discusión. Isabel Cecilia Martínez nos dice al respecto:

"El modelo que adoptamos fue distinto al de las sociedades que había en la Argentina, que no eran específicamente de investigación. A nosotros nos parecía mejor tratar de dar un formato similar al que conocimos en esas primeras conferencias y que tenía que ver más con una concentración no tanto en las personas sino en los trabajos, es decir en la línea del trabajo, el contenido del trabajo, la temática del trabajo y no tanto en quién era el que lo presentaba. En ese momento era más importante la persona que los trabajos."

Así confluyeron en las primeras reuniones el encuentro de colegas investigadores, el interés en la experiencia musical como objeto de estudio y el nuevo formato que los congregaba y organizaba; contando además con la asistencia de investigadores internacionales especializados en psicología de la música que, de alguna manera, apadrinaron los inicios de la Sociedad. Asistimos desde entonces 
a una paulatina consolidación del organismo que hoy alcanza una fuerte impronta local y regional:

"La primera reunión fue en Avellaneda donde estaba la carrera de musicoterapia. La idea era la de hacer una conferencia relativamente chica, que durase un día, que reuniera pocos trabajos y que sirviera para discutirlos. Planteamos esa primera reunión en la facultad de Psicología de la UBA y vino como invitado Michel Imberty. Se reunieron una decena de trabajos y se hizo en un día. La verdad es que estuvo muy lindo, vino mucha gente y el lugar se llenó. Me acuerdo que nos sorprendió. Ahí mismo anunciamos que la 2da Reunión iba a ser en Quilmes, donde trabajaba Orlando Musumeci. Entonces empezó la costumbre de anunciar en cada reunión, dónde se haría la siguiente." Relata Favio Shifres.

"Me acuerdo de la segunda reunión que fue en Quilmes, vino John Sloboda de conferencista invitado. Nos dijo: para poder desarrollar las ciencias cognitivas de la música o desarrollar la investigación, tienen que crear carreras de posgrado. Al hacer un posgrado, la persona empieza a estudiar y para escribir su tesis, tiene que investigar. Así es como se va a desarrollar el campo. Fue un momento que yo recuerdo como de mucha ebullición. Nos hacíamos preguntas respecto de lo que estábamos haciendo en la formación musical en la universidad, preguntas que no tenían respuesta a partir sólo de los marcos teóricos que estábamos manejando en ese momento. Entonces nos dio mucha curiosidad acerca de la experiencia musical.” Palabras de Isabel Cecilia Martínez.

En la entrevista recordamos luego una tercera reunión en el rectorado de la UNLP, a la cual asistieron Ian Cross desde Inglaterra y Michel Imberty desde Francia. Allí tuvo lugar la presentación de trabajos originales y un interesante debate sobre los temas culturales y educativos. Hubo también una gran concurrencia de colegas y de estudiantes desde diferentes lugares de la región y Europa.

Producto de la reflexión permanente que ha acompañado cada etapa del proceso de investigación, aparecieron los primeros cambios en el estudio de los temas centrales relacionados a la experiencia musical, así como su aplicación en otras áreas de conocimiento y sus vinculaciones con diferentes ciencias; aspectos todos que le dan identidad a SACCoM como sociedad, y que a su vez impactan en nuestra región. Favio Shifres nos lo cuenta de esta manera:

"Creo que la Sociedad fue importante en determinar una posibilidad de desarrollo del conocimiento. Hasta ese momento no existía, en el país ni en toda la región. No existían, por lo tanto, las referencias al problema de qué es la experiencia musical, cómo se vincula con la construcción del conocimiento, cómo se vincula con áreas de aplicación -entre ellas la educación musical, la inteligencia artificial- cómo se 
vincula con áreas de conocimiento básico como las neurociencias, la acústica, etc. Y no existía cómo se relaciona la experiencia musical con las formas de pensar la música y las disciplinas vinculadas como la musicología, la etnomusicología y todo ese entramado. Las referencias que había con relación a esto se identificaban con las cosas que se hacían en otro lugar de acuerdo con las cosmovisiones de ciencias y de abordaje de estudio de esos lugares, pero no había un abordaje que atendiera las necesidades locales. Creo que todavía no existe, pero hemos hecho un caminito hacia eso. Me parece que la creación de la Sociedad ha sido importante para abrir esa puerta. El campo de los estudios en música en general está muy compartimentado, algo con lo que nosotros tratamos de luchar desde el principio. Me parece que la Sociedad tuvo cierta repercusión en la región porque en otros países empiezan a surgir sociedades similares, se empieza a incorporar un poco de investigación en las universidades y me parece que la Sociedad tuvo algo que ver en todo esto."

Siguiendo con el relato, Isabel Cecilia Martínez recuerda:

"Viéndolo así a través de los años, creo que el impacto ha sido positivo en general en Argentina, independientemente de que el campo siga concentrado en algunos focos determinados de investigación. Además, se extendió primero a Brasil, es decir, la Sociedad Brasilera de Cognición y Artes Musicales (Associação Brasileira de Cognição e Artes Musicais) se desarrolló a partir de la asistencia de investigadores brasileños a nuestras reuniones, tomando gran impulso. Después algunos de nosotros tuvimos un desarrollo de trabajo personal. Viajamos durante casi 10 años a Colombia donde se formó otro núcleo.”

La Sociedad ha sabido construir huellas al incluir diferentes actores de la vida musical argentina y latinoamericana luego de recorridos sus 20 años. Entiende de logros significativos como es el acceso libre al conocimiento compartiendo papers desde sus inicios a través de su sitio web, la apertura a la interacción entre los distintos campos interdisciplinarios, y la creación del LEEM, un laboratorio específico para el estudio de la experiencia musical en la Universidad Nacional de La Plata:

"Hicimos algunas cosas que fueron interesantes, como instalar la idea de que una conferencia empieza a una hora, un trabajo dura un determinado tiempo, después de ese tiempo viene una discusión de un determinado tiempo y después viene otro trabajo que dura ese mismo tiempo. Estas cosas no sucedían en las conferencias en nuestro país. Esas maneras, que, aunque parezcan superficiales, sumaban para concentrarse en el contenido de los trabajos, que era lo más importante. Había un protocolo para hacer conferencias, los espacios tenían que ser amplios, luminosos, debían permitir la circulación de la gente. Era importante que la gente tuviera la 
libertad de ir de un lugar a otro, sin perderse los trabajos. Se consideraban ciertas cuestiones relativas al respeto hacia el asistente a la conferencia, hacia su tiempo que también valía. Una cantidad de detalles que ahora nos parecen tontos, pero que en realidad fueron importantes para establecer una manera de plantarse frente a la difusión de la investigación. Quizás en otras disciplinas estos modos estuvieran instalados, pero nosotros en la música no lo teníamos. Es decir, esta idea de que los trabajos debían tener fuentes, las fuentes tenían que ser consultadas, tenían que ser citadas de determinada manera, con determinados procedimientos, la idea de que el conocimiento se compartía..."

En palabras de Isabel Cecilia Martínez, a lo que agrega:

"Hay que tener en cuenta que cuando organizamos el sitio web de la Sociedad, los trabajos de las conferencias se fueron subiendo y desde el primer momento quedaron accesibles para todas las personas que quisieran consultar. Hoy los trabajos de nuestra Sociedad son una fuente de consulta muy amplia. Si bien algunos de nuestros propósitos iniciales eran utópicos ya que, los núcleos de investigación en psicología de la música en veinte años no son los que imaginábamos en el inicio, sin embargo, el tema resulta de interés y aspectos de la experiencia musical se han ido incorporando en los planes de estudio de carreras de grado y posgrado en diferentes instituciones universitarias y terciarias de formación musical en el país y en Latinoamérica. Con relación a nuestro grupo de trabajo en la UNLP, la creación del Laboratorio para el Estudio de la Experiencia Musical es uno de esos logros. En ese sentido, creo que fuimos pioneros en eso del acceso abierto al conocimiento. Ese fue nuestro interés: que el conocimiento sea accesible a todo el mundo. Y eso creo, es una cosa muy importante que hizo SACCoM desde el origen y que sigue haciéndolo, por supuesto."

Resulta evidente que la Sociedad sostiene como rasgo esencial la idea de construir conocimiento y compartirlo en el marco del encuentro entre las personas, de manera presencial y virtual, y trascendiendo incluso las fronteras.

"Sí, eso fue lo que tratamos de consolidar desde el principio. Que el encuentro fuese un encuentro siempre constructivo. Desde el comienzo la idea fue construir comunidad. Construir conocimiento a partir de construir comunidad. Por eso siempre tuvimos la idea de modificar los lugares de realización de los encuentros, para favorecer el intercambio y que la Sociedad fuese un lugar donde la gente se pudiera encontrar. Hicimos muchas cosas: tuvimos propuestas virtuales, entre ellas la publicación de la revista Epistemus que es de amplísima convocatoria. Recibe trabajos de una diversidad temática y conceptual muy grande porque el problema de estudiar la experiencia musical es un problema que puede ser visto desde múl- 
tiples perspectivas, entonces eso acepta la reunión de intereses muy muy diversos. $\mathrm{Y}$ yo creo que esto también es lo que hemos ido haciendo nosotros en el trabajo específico como investigadores. Individualmente y por fuera de la Sociedad. Generar equipo donde hay músicos trabajando con psicólogos, con filósofos, hemos trabajado con biólogos, con computólogos, digamos con gente que desde diversos lugares se han interesado por problemas de la música y de la relación con y entre las personas y la música", relata Favio Shifres.

Otro rasgo importante de la Sociedad es la capitalización de los diversos intereses, edades y personas que se acercan a la institución y el compromiso que esto despierta en la formación de los recursos humanos en la especificidad del investigador, como otro canal posible de abordar la práctica/estudio de la música. Favio Shifres nos da su parecer:

"Lo que digo siempre es que hay que trabajar con la gente que se está formando. Por eso son importantes las instancias de posgrado y las becas de posgrado. Es necesario que la gente tenga dentro de los horizontes la perspectiva de dedicarse a esto, si no, nadie lo va a hacer. Digamos, los estudiantes entran a la universidad a estudiar música, o a estudiar psicología, o a estudiar filosofía, o cualquier disciplina vinculada con la música, pero no piensan que inicialmente pueden estudiar el aspecto de la experiencia musical entendido como aquello que el ser humano experimenta en el involucramiento en la música. El que va a estudiar música piensa que su horizonte de expectativa es subir al escenario y tocar, o componer, o lo que sea. El que va a estudiar psicología, piensa que su horizonte de expectativa es, atender pacientes, o preocuparse por procesos generales de conocimiento. Y así, con cada cosa. ¿Qué quiere decir? Que este campo de conocimiento está en una intersección, pero al mismo tiempo está como oculto. Entonces si no se conoce, a nadie se le ocurre. Lo digo porque tengo experiencia de enseñar en posgrados que no son de música. Al finalizar el curso de Psicología de la música se me acerca gente que nunca había pensado en las cosas de las que hablamos ahí. Y gente que son psicólogos, antropólogos o filósofos, vienen y me dicen: che, vos sabés que yo desde cbico toco tal instrumento, soy músico, tengo una banda y nunca habia vinculado nada de eso con mi profesión. Entonces empiezan a pensar su propio campo de conocimiento en la psicología, la antropología, la filosofía, desde esa experiencia musical de vida que tienen y se dan cuenta que hay un espacio disciplinar para que ese encuentro tenga un lugar de indagación sistemática."

\section{Palabra finales}

El encuentro con Favio e Isabel nos ha dejado un valioso testimonio acerca 
de la creación de la Sociedad y sus ideas fundacionales. Fueron evocados los principios que le otorgan identidad y originalidad al estudio de la experiencia musical, desarrollada en los contextos socioculturales en los que tiene lugar desde una perspectiva plural e interdisciplinar.

La Sociedad Argentina para las Ciencias Cognitivas de la Música es un espacio abierto y en construcción permanente desde donde se alienta de manera constante a los distintos actores de la vida musical argentina y latinoamericana a unirse, a acercar sus proyectos, sus ideas, sugerencias e inquietudes. SACCoM es de todos lo que quieran formar parte de ella.

\section{Notas}

1. Mirian Tuñez: http://orcid.org/0000-0002-1560-1488

2. Guadalupe Segalerba: http://orcid.org/0000-0002-1390-7897 